\title{
Two-dimensional movement patterns of vibratory conveyors
}

\author{
PROF. DR.-ING. KLAUS NENDEL \\ DiPL.-ING. THOMAS RISCH \\ CHEMNITZ UNIVERSITY OF TECHNOLOGY \\ FACULTY OF MECHANICAL ENGINEERING \\ PROFESSORSHIP CONVEYORS
}

Vibrationsförderer stellen sowohl für Schüttgüter als auch für Stückgüter kleiner bis mittlerer Abmessungen einen wesentlichen Anteil der eingesetzten Zuführtechnik dar. Die dabei vom Fördergut erreichten Fördergeschwindigkeiten sind maßgeblich vom Betriebspunkt eines Vibrationsförderers abhängig und werden in der Praxis häufig durch die Betriebsfrequenz, die Schwingweite und den Wurfwinkel zur Horizontalen bestimmt. Der Einsatz von Schwingungsisolatoren zur Minderung der an die Umgebung übertragenen dynamischen Kräfte kann am Förderorgan Bewegungsformen verursachen, welche die Berechnung der resultierenden Fördergeschwindigkeit nach den bekannten Richtlinien nicht mehr ermöglicht. Anhand theoretischer und experimenteller Untersuchungen werden Analysemethoden zur Bestimmung zweidimensionaler Bewegungsformen vorgestellt und weiterführende Ansätze zur Simulation der Fördergutbewegung angeschnitten.

Vibratory conveyors represent an essential proportion of the feeding technology used for small and medium-sized bulk goods and piece goods. The obtained speed of the conveyed good is considerably dependent on the operating point of a vibratory conveyor and is often determined by the operating frequency, the amplitude and the throwing angle to the horizontal. The use of vibration isolators to reduce the dynamism transferred to the environment may cause movement patterns of the conveyor that make the calculation of the resulting conveying speed according to the known guidelines impossible. Analysis methods of two-dimensional movement patterns are introduced with the help of theoretical and experimental examinations. Further approaches to simulate movement patterns of conveyed goods are introduced.

\section{Introduction}

Oscillating or vibratory conveying technology is a special field of conveyor technology in the area of continuous conveying processes. The demands on the used technology are related to short and medium distance conveying (mainly distances of less than 10 metres) as well as to the sorting, positioning and dosage of goods.

Concerning bulk goods with mainly granular characteristics, the main focus is on the conveying of goods that takes place within associated processing or refinement processes like sorting or drying. In the case of small and medium-sized piece goods that are in an unorganised way within bunker units, oscillating conveying systems are used in order to bring the piece goods into a defined position for further transportation and subsequent processing. Necessary material, energetic and technical costs are very low compared to the use of other conveying or handling systems like sensory controlled circulating traction mechanisms or operated gripper systems (robotics). Vibratory conveyors until today make up a share of about 80 percent of all used conveying technology due to their numerous advantages like simple technical construction, high reliability and almost wear-free operational behaviour.

\section{Theoretical foundations}

\subsection{Construction, mode of action and classification}

Basically, the construction of vibratory conveyors is characterised by a supported conveyor organ capable of oscillation. It consists of a channel (conveyor channel), tube or tray that is put into a periodical bidirectional movement by an initialising force or a set path . This movement is called operating point and is characterised by the frequency, the amplitude (double maximum value of the movement amplitude) and the angle between the horizontal and the direction of motion of the conveyor organ. 

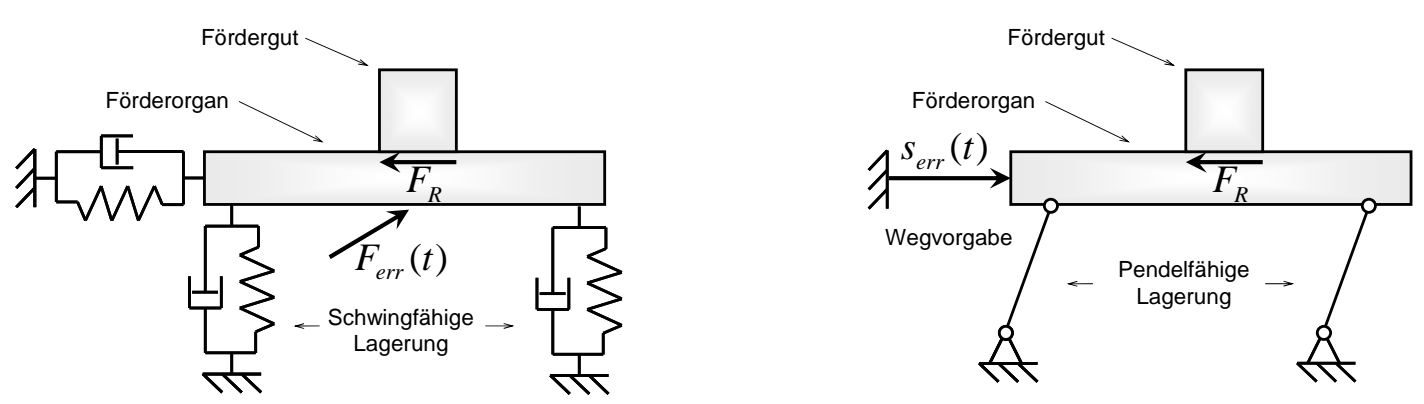

Figure 1: Generalised construction of an oscillated conveyor; Oscillating system (left); Mechanism (right)

The conveyor organ accelerates the good placed on it into the conveying direction via frictional forces on the contact areas. Despite a backward movement of the conveyor organ, the conveyed good remains in the original conveying direction due to its mass inertia. If the relative movement of the conveyed good to the conveyor organ is higher in one direction within a motion period, a movement of the conveyed good will result. The modulus of this movement is called conveying speed.

Within the micro throw principle, the movement pattern of the conveyor organ forces the conveyed good into a micro throwing movement. In the simplest case, the conveyor organ moves with a throwing angle $\beta$ to the horizontal, a operating frequency $f_{b}$ and an amplitude $2 \hat{q}$. A vertical acceleration part results from the throwing angle $\beta$. If the downward aimed vertical acceleration part of the conveyor organ exceeds the acceleration due to gravity $g$, the conveying good begins to lift off and is thrown. The good remains in the original direction of motion due to its mass inertia and moves further on a micro throw parabola in the conveying direction. Almost all oscillating conveyors used for conveyance on the horizontal level or with a small gradient angle work with the micro throw principle.

The slip principle is characterised by the permanent contact between conveyed good and conveyor organ. Therefore, the downward aimed vertical acceleration part must never exceed the acceleration due to gravity during the movement of the conveyor organ. A relative movement of the conveyor organ is still possible, if the normal force component of the good shows different moduli during back and forth hub. Beside a few special exceptions, the slip principle is almost exclusively realised by oscillating conveyors built as vibrating feeding chutes.

\subsection{Calculation of conveying speed}

In order to integrate oscillating conveyor devices into production lines, it is essential to know the operational capacity of the conveyed good like volume or mass flow. The increasing demand for logistically efficiently synchronised parts of the production flow leaves only little scope in which the conveying performance may vary. Adaptations of the throughput rate of goods can only be implemented, if the operating point of already integrated devices can subsequently be modified as required. It is essential for the dimensioning that the constructing engineer is provided with exact calculation regulations in order to calculate the expected conveying speed $v_{F}$ of the conveyed good flow.

In the history of development of oscillating conveyor technology, numerous differently detailed analytical models were introduced that described the movement of the good during the conveying process. In practice, to calculate the theoretical conveying speed, the calculation rule included in the VDI 2333 has prevailed [VDI.65]. This calculation rule is based on models developed by Böttcher and Wehmeier in the 1970s and is the result of an analytic solution to the formulated problem.

$v_{t h}=\frac{g \cdot \bar{n}^{2}}{2 f_{B}} \cdot \cot \beta$

Advantages of this calculation rule are the simplicity and the good practical suitability for values of a relative throwing time $\bar{n}=0,8 \ldots 0,9$. Simplifications and idealised assumptions made during modelling, however, considerably limit the information spectrum of this calculation rule. The rule can only be used on the assumption 
of a harmonic (sinusoidal) movement of the conveyor organ. It is provided that the good only jumps within a period and that it has idealised grip characteristics. Impulse effects and relative movement to the conveyor organ are totally ruled out. Grip and slip friction forces between the conveyed good and the conveyor organ are also neglected. Furthermore, this model does not provide information about slip conveyance because the calculation of the theoretical conveying speed is dependent on a relative throwing time component. Within the slip conveyance principle, this component is zero.

In many cases, all these limitations in favour of an analytic solution do not allow to comprehensively describe the complex dynamic processes during an oscillating conveyance process. Especially in those cases, where the movement of the conveyor organ differs substantially from a harmonic, linear path, there will be significant differences between the calculated and actually observed conveying speed. Smaller oscillating conveyors like transportation and assembly technology are an example here.

\section{2D movement patterns of vibratory conveyors}

\subsection{Causes of 2D movement patterns}

There are several causes for the emergence of two-dimensional oscillation patterns of vibratory conveyors. They can be generated intentionally or unintentionally. Especially in the case of smaller oscillating conveying devices in the field of transportation and assembly technology, the thereby caused effects on the conveying process become apparent.

The forces transferred from vibratory conveyors to the environment in many cases have to be minimised in order not to influence the functionality of peripheral devices. Thus, the principles of mass and torque balance as well as the principle of oscillation isolation are implemented. Oscillation isolators, however, also function as additional spring elements that substantially influence the dynamic characteristics of the overall spring-mass system of oscillating conveyors.
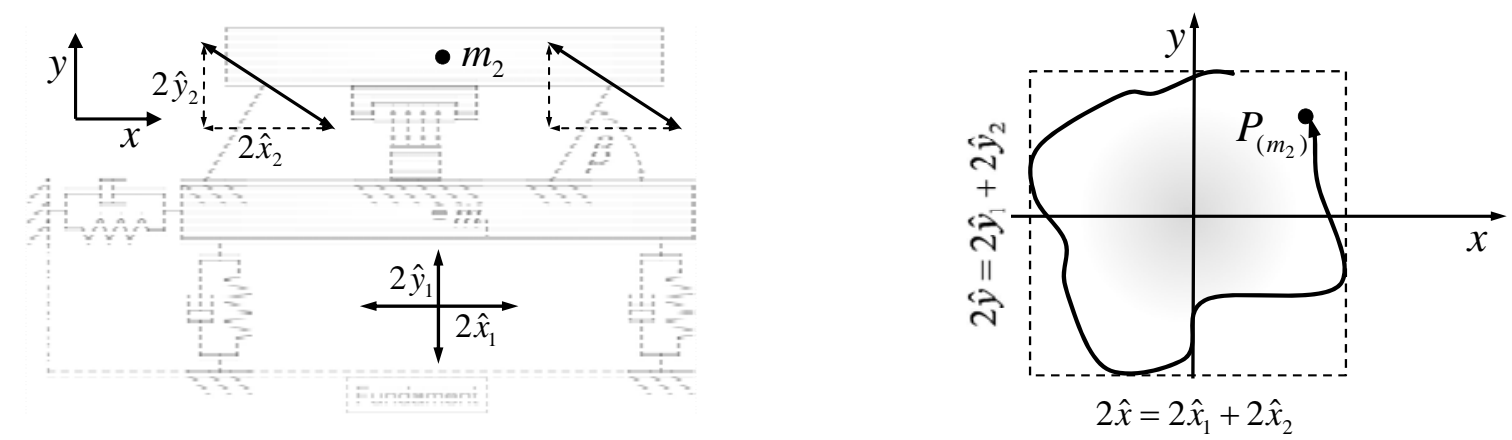

Figure 2: Degree of freedom of an oscillation isolated vibratory conveyor (left); any two-dimensional movement pattern (trajectory) of the conveyor organ (right)

In addition to the freedom of motion in $\mathrm{x}$-direction, the partly masses have also vertical degrees of freedom. These result from the spring mounting of the rack frame $m_{1}$ in y-direction. In practice, this degree of freedom of movement is mostly neglected because the oscillation amplitudes of the rack frame in vertical direction are mostly essentially lower than the amplitudes in conveying direction. But in particular this vertical degree of freedom often is the cause for the emergence of two-dimensional movement patterns of the conveyor organ and may considerably influence the conveying process.

The position of the conveyor organ $P_{\left(m_{2}\right)}$ can be described by two coordinates $x(t)$ and $y(t)$ at every point in time. The path of $P_{\left(m_{2}\right)}$ is called trajectory or movement pattern. In Figure 2, any two-dimensional movement pattern is shown. In principle, the trajectory may have any possible course, but always has to be closed for the oscillating conveying process is a periodic process. It corresponds to the oscillation answer of the overall system and can be illustrated as overlapping of the partial oscillation components in the respective coordinates' direction according to the superposition principle. 


\subsection{Description of harmonic movement patterns}

From the periodicity of the oscillating conveying process follows that the absolute coordinates form pairs of variates which temporally recur with a certain frequency. This means that in any case a closed trajectory $y(x)$ is formed by an overlapping of both movement components. A first order harmonic movement pattern is formed if the conveyor organ sinusoidally (harmonically) moves in both vertical and horizontal direction. If both movement components reach their deflection maximum or deflection minimum not simultaneously, one speaks of a phase shift termed as $\varphi$.
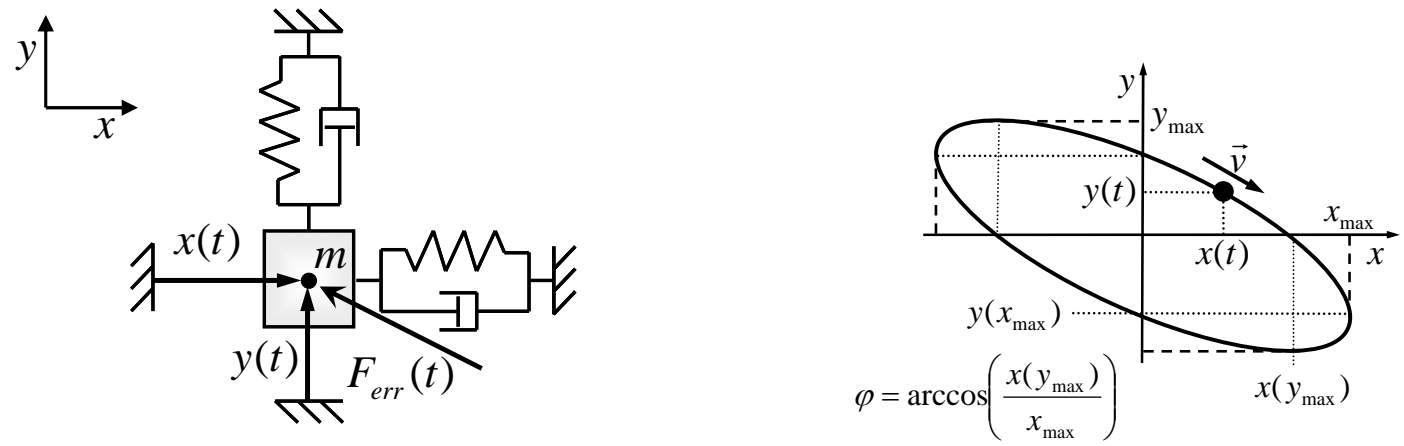

Figure 3: First order harmonic movement pattern as result of the superposition of a vertical and a horizontal partial oscillation component; a phase shift leads to a elliptic movement pattern

First order harmonic movement patterns technically can relatively easy be generated since they correspond to the oscillation answer of linear spring-mass systems. If the damping of spring-damper elements is neglected during modelling, only phase shifts of $\varphi=0$ or $\varphi=\pi$ may occur. The mass in figure 3 oscillates in any case with the frequency of initiation. Dependent on the adjustment of the drafted spring elements as hypercritical or subcritical towards mass $m$, the oscillation answer is either accurately in phase or accurately out of phase to initiation.

If the damping is not neglected, any phase shift between $0<\varphi<2 \pi$ is possible and may be generated by the appropriate choose of the rigidity of the horizontal and vertical spring elements.

By using conventional flat spring or rubber spring elements, placed horizontally and vertically, and simple drive units that initiate partial oscillations in both directions, two dimensional movement patterns can deliberately be generated. Those movement patterns often accidentally occur with many oscillating conveyors, often remain undetected, or they are neglected during the calculation of the conveying speed. This often leads to considerable deviations of the calculation results of the expected conveying speed.

Two-dimensional movement patterns may take very complex forms that cannot be sufficiently described by overlapping two harmonic functions. Nevertheless, each periodic signal can be described as an infinite series of harmonic or trigonometric functions. This characteristic is used to formulate a generalised possibility to describe $k$-order movement patterns.

$$
\begin{aligned}
& x(t)=\sum_{n=0}^{k} C_{x n} \cdot \cos \left(n \Omega t+\varphi_{x n}\right) \\
& y(t)=\sum_{n=0}^{k} C_{y n} \cdot \cos \left(n \Omega t+\varphi_{y n}\right)
\end{aligned}
$$

The interpretation of movement patterns as trigonometric series show numerous advantages with regard to further examinations. Almost every imaginable trajectory may be designed whereas very good approximations to experimentally achieved oscillation graphs are possible, even with a small number of series elements $(k \leq 5)$. Experimental data can be reproduced in form of a coefficient matrix and documented in a simple database. Furthermore, trigonometric series are characterised by continuity and differentiability, and thus the series are suitable as input functions of numeric simulation programmes.

$\stackrel{(n)}{x(t)}=\frac{d^{n} x}{d t^{n}}=\sum_{n=0}^{k} C_{x n} \cdot n \Omega^{n} \cdot \frac{d^{n}\left(\cos \left(n \Omega t+\varphi_{x n}\right)\right)}{d t^{n}}$ 


\section{Experimental examinations}

The following test set-up was designed in order to experimentally examine the consequences of two-dimensional movement patterns on the oscillating conveying process. Several vibratory conveyors with two-dimensional movement patterns on the conveyor organ were examined in operating state with the help of laser triangulation sensors. At the same time, horizontal and vertical path-time processes as well as the movement behaviour of the conveyed good were recorded. Due to the joint time basis of the measuring signals, it is possible to interpret the reaction of the conveyed good to certain movement patterns.

The vertical movement of the conveyor organ was recorded at two points (on the left and right side) to evaluate the steadiness of the movement pattern over the entire length of the conveyor organ. By this methodology, staggering or tilting movements of the conveyor organ can be detected, and conclusions on suitable measuring points can be drawn. In addition to the metrological examinations via laser triangulation, high-speed recordings of the movement of the conveyed good were made. On the one hand, these recordings should visually support the sensor signals. On the other hand, they should help to model a suitable algorithm for the description of the conveying process.
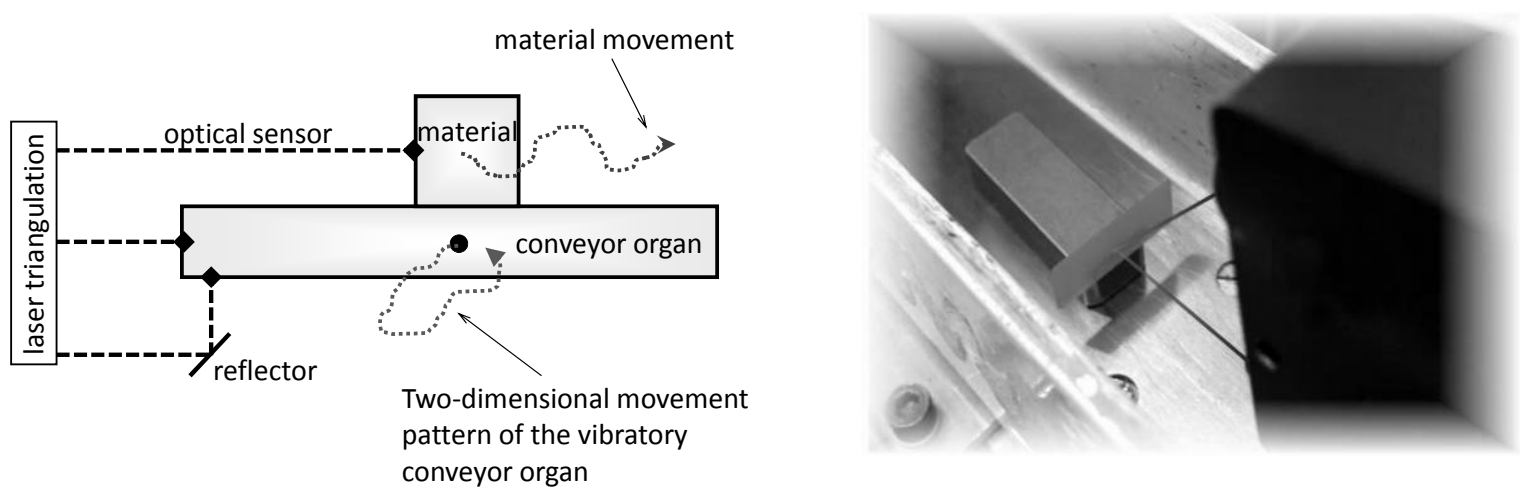

Figure 4: Test set-up to examine the movement pattern via laser triangulation (left); recording the reaction of the conveyed good (right)

The average rise of the course $p(t)$ in figure 5 correlates with the medium conveying speed of the good as reaction to the $2 \mathrm{D}$ movement pattern of the conveyor organ. The chosen measured values are characterised by an operating frequency of $50 \mathrm{~Hz}$, an amplitude of $0.8 \mathrm{~mm}$ and a resulting medium conveying speed of about 2.7 $\mathrm{m} / \mathrm{min}$.
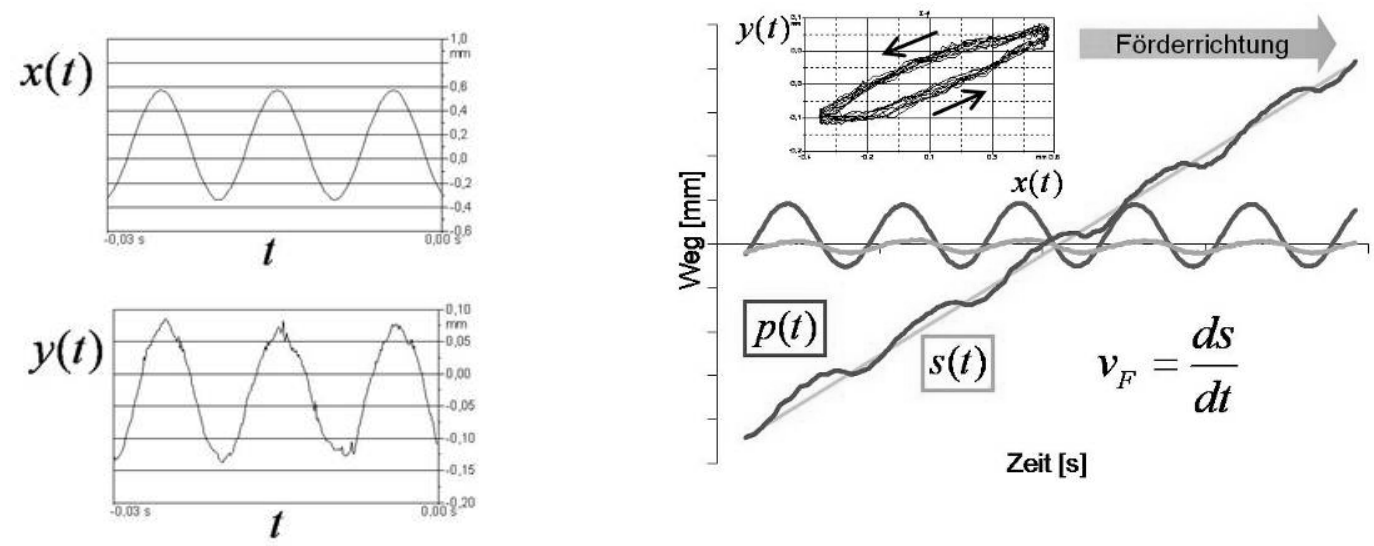

Figure 5: Exemplary measurement: chosen sensor signals of conveyor organ (left); overlapping of sensor signals on a joint time basis, identification of relevant values (right)

The recorded sensor data was analysed with discrete Fourier transformation and a specially developed macro programme and documented in form of a coefficient matrix. This data structure forms the starting point for the verification of a still developing mathematic model for the time-discrete calculation of the behaviour of a conveyed good with two-dimensional movement pattern. Single verifications were already made and showed that the calculated medium conveying speeds on average differed from the experimentally required data by less than $10 \%$. 


\section{Summary and preview}

At the Chemnitz University of Technology, Faculty of Mechanical Engineering, Professorship Conveyors, several vibratory conveyors with $2 \mathrm{D}$ movement patterns of the conveyor organ at the operational point were experimentally examined. At the same time, the movement pattern of the conveyor organ and the consequentially resulting movement of the conveyed good were contact-freely recorded by laser triangulation sensors. The path-time processes got by discrete sensor signals are analysed with the help of discrete Fourier analysis, reproduced in form of a Fourier coefficient matrix and documented in a database. This experimental data is used for the empirical interpretation as well as for the verification and enhancement of a specially developed mathematic model. On the basis of this mathematic model, a simulation tool should be developed, which will help to calculate conveying speeds more precisely and more detailed than established calculation rules according to the VDI guideline are able to. At the same time, optimal movement rules for oscillating conveyors can be derived. They consequently allow the deliberate development and constructive implementation of efficient vibratory conveyors.

\section{Literature}

[Trö.06]

[Opp.04]

[Bri.95]

[Ben.05]

[VDI.65]

[Böt.57]

[Weh.61]

[Hab.84]

[Der.06]
Trölß, J.: Komplexe Zahlen und Funktionen, Vektoralgebra und analytische Geometrie, Matrizenrechnung, Vektoranalysis. Wien, New York: Springer-Verlag, 2006. ISBN 3-211-29687-5.

Oppenheim, A. V.; Schafer,R. W.: Zeitdiskrete Signalverarbeitung. 2. überarbeitete Auflage [Neuübers.]. München, u. a.: Pearson Studium, 2004. ISBN 3-8273-7077-9.

Brigham, E. O.: Schnelle Fourier-Transformation / übers. Von Seyed Ali Azizi. 6., korrigierte Auflage. München, Wien: Oldenbourg Verlag, 1995. ISBN 3-486-231774.

Benker, H.: Differentialgleichungen mit Mathcad und Matlab. 1. Auflage. Berlin, Heidelberg [u.a.]: Springer Verlag, 2005. ISBN 3-540-23440-3.

VDI-Richtlinie 2333. Schwingförderer für Schüttgut. Ausg. 10. 1965.

Böttcher, S.: Beitrag zur Klärung der Gutbewegung auf Schwingrinnen. Hannover: Diss. Technische Hochschule Hannover, 1957.

Wehmeier, K.-H.: Untersuchungen zum Fördervorgang auf Schwingrinnen. Hannover: Diss. Technische Hochschule Hannover, 1961.

Habenicht, D.: Grundlagenuntersuchungen zur Werkstückgleitförderung in Schwingzuführsystemen. Hannover: Diss. TU Hannover, 1984.

Dresig, H.; Holzweißig, F.: Maschinendynamik. 7., bearbeitete Auflage. Berlin, Heidelberg: Springer-Verlag, 2006. 